\title{
Randomized Clustering Scheme for Heterogeneous Wireless Sensor Networks
}

\author{
Ahmed Salim \\ Math. Dept., Fac. of Sci., Univ. of Zagazig, Zagazig, P. O. Box 44519, Egypt. Math. Dept., \\ Fac. of Sci. and Arts, Al-mithnab, Qassim Univ., P. O. Box 931, Buridah 51931, \\ Al-mithnab, Kingdom of Saudi Arabia.
}

\begin{abstract}
Wireless Sensor Networks (WSNs) are resource-constrained systems. Efficient use of resources especially, energy is most important for their lifetime extension. Clustering of sensor nodes is a well-known approach for achieving high scalability and efficient resource allocation in WSN. We propose a dynamic, distributive, and self-organizing algorithm that utilizes a simplified clustering approach to organizing the WSN into two-level of the hierarchical network. We consider three-level energy heterogeneity of sensor nodes and takes the advantage of the local information such as residual energy, a number of neighbors and distance to the base station as criteria for $\mathrm{CH}$ election and cluster formation. Simulation results show that compared with the existing three-level energy heterogeneity based clustering algorithms, our algorithm can achieve longer sensor network lifetime.
\end{abstract}

\section{Keywords}

Wireless sensor networks, Clustering, self-organizing, distributive, three-level energy heterogeneity

\section{INTRODUCTION}

Todays, WSNs has been an important domain in computer science and technologies and has wide applications in the future. It has enabled the development of a sensor node with a minimum cost processor, low power, and lightweight. A WSN consist of sensor nodes, each node has small sensor able to detect a lot of things (such as light, sound, temperature, motion, etc.). This node has also an intelligent computing device that enables the processing of data that collected from sensors, and it has wireless communication capabilities. Sensor nodes using their wireless interfaces to organize networks and communicate with each other. In large-scale sensor networks, hundreds or thousands of sensor nodes are randomly deployed into a sensing field [1] [2].

Clustering algorithms in WSN apply data aggregation techniques which reduce the collected data at cluster head in the form of significant information [3, 11]. Cluster heads then send the aggregated data to Base Station (BS).

Most of the clustering algorithms for WSNs can be classified into two main categories, depending on cluster formation criteria and parameters used for $\mathrm{CH}$ election, Probabilistic (random or hybrid) clustering algorithms and deterministic clustering algorithms [15].

In probabilistic clustering algorithms, each sensor node has a priori probability to determine the initial $\mathrm{CHs}$. On the other hand, deterministic clustering algorithms based on special attributes of the sensor node such as, connectivity, degree, etc., and on the information received from other closely located nodes[15].

In this paper, an energy-efficient clustering scheme that falls between the two categories of clustering algorithms, randomize clustering algorithms and deterministic clustering algorithms. This scheme uses both randomize as well as a deterministic approach to achieve better energy efficiency.

The proposed scheme uses randomized sensor nodes' proximity (residual energy, distance to base station and neighbors' density) as the election criterion. Simulation results show that the proposed algorithm is able to manage energy consumption better and achieves the desired result for wireless sensor networks.

The rest of the paper is organized as follows: Section 2 briefly reviews related work. The energy model is described in Section 3. In Section 4, our scheme will be introduced. Section 5, the simulation results of our approach is presented. In Section 6, we conclude our work.

\section{RELATED WORK}

WSNs have attracted much attention in recent years due to their potential use in several applications such as healthcare monitoring system, forest fire, and border surveillance applications [1, 2].

LEACH [5] is the first and most popular energy-efficient hierarchical clustering algorithm for WSNs that was proposed for reducing power consumption. One of the key features of LEACH is a randomized rotation of the Cluster Head $(\mathrm{CH})$ and corresponding clusters. LEACH divided into two phases, setup phase, and steady state phase. Sensor nodes in setup phase select themselves to be $\mathrm{CHs}$ at any given round with a certain probability. Each sensor node that is not a $\mathrm{CH}$ selects the closest $\mathrm{CH}$ and joins that cluster. The $\mathrm{CH}$ then creates a schedule for each cluster member node to transmit its data. In steady state phase, the cluster member node transmits the data to the $\mathrm{CH}$ in their assigned time slot.

LEACH-Centralized (LEACH-C) [6] is similar to LEACH, however, LEACH-C uses a centralized algorithm to select the $\mathrm{CH}$ (i.e. the BS determines which node will become the $\mathrm{CH}$ ). The work in [4] propose optimization Low Energy Adaptive Clustering Hierarchy (O-LEACH). O-LEACH improve LEACH and LEACH-C by selecting cluster according to the residual energy of nodes dynamically. The authors in [4] concluding that O-LEACH increases the stability of the network and it can be used for dynamic networks.

Low Energy-Adaptive Clustering Hierarchical Protocol with a Deterministic cluster-head (LEACH-D) [7] similar to the original LEACH except that they considered additional component which takes into account both the probability $\mathrm{P}$ of election as cluster-head and the residual energy of each node. In [8], the authors proposed Deterministic Energy-efficient Clustering protocol (DEC) which uses residual energy to elect $\mathrm{CHs}$ in a deterministic way but outperforming than the probabilistic-based protocol LEACH.

In [11], the authors take several node attributes into consideration, such as node degree, transmission power, mobility, and battery power. These parameters are weighed 
correspondingly and summarized to get a combined weight. The node with the smallest weight is chosen to be a $\mathrm{CH}$. This weighted clustering algorithm (WCA) is completely distributed and dynamic so that it quite adapts to the everchanging topology.

The authors in [12] proposed an energy heterogeneous-aware protocol called Stable Election Protocol (SEP). SEP is based on weighted election probabilities of each node to become cluster-head according to the remaining energy in each node. In their work, they use a two node energy classification to characterize the energy heterogeneity problem in the network. They further adapted the weighted probabilities according to the types of nodes in the network. Specifically, they labeled some nodes as 'normal nodes' and some nodes as 'advanced nodes'. Each type of node elects itself according to new sets of the threshold. The work in [13] proposed extended heterogeneous LEACH (EHE-LEACH) protocol that is used the same model in SEP [12].

The Distributed Energy- Efficient Clustering Protocol (DEEC) [10], following the same principle of energyheterogeneity of SEP. The DEEC protocol elects cluster-heads based on the ratio between the residual energy of each node and the average energy of the network. The epochs of being cluster-heads for the nodes are different according to their initial and the residual energies.

The work in [9] proposed SEP extension called SEP-E, by considering a three-level energy heterogeneity of sensor node in a two-level hierarchical network similar to the energy model used in DEC. In our work, we use the three-level energy heterogeneity of sensor node as in DEC and SEP-E.

\section{ENERGY MODEL}

We use the same data aggregation model, energy parameters, and radio model as discussed and used in a number of previous studies $[12,8,10,5]$, wherein the energy consumption is mainly divided into two parts: receiving and sending messages. The sending energy consumption needs additional energy to enlarge the signal depending on the distance to the destination. Thus, to transmit a $l$-bit message a distance $\mathrm{d}$, the radio power consumption will be,

$$
E_{T x}(l, d)=\left\{\begin{array}{cc}
l E_{\text {elec }}+l \epsilon_{f s} d^{2} & \mathrm{~d}<d_{0} \\
l E_{\text {elec }}+l \epsilon_{m p} d^{4} & \mathrm{~d} \geq d_{0}
\end{array}\right.
$$

and to receive this message, the radio expands will be

$$
E_{R x}(l)=l E_{\text {elec }}
$$

where $d$ is the distance between sender and receiver, $E_{T x}(l, d)$ is the cost of transmitting an $l$-bit message for a distance $\mathrm{d}, E_{R x}(l)$ is the cost of receiving and $l$-bit message for a distance d, $E_{\text {elec }}$ is the electronics energy that depends on the circuit itself, $\epsilon_{m p}$ (amplification energy expended to overcome the multi-path) is the energy consumed by the transmitter amplifier for longer distance, $\epsilon_{f s}$ (amplification energy expended to overcome the free space) the energy consumed by the transmitter for shorter distance, the threshold distance $d_{0}=\sqrt{\frac{\epsilon_{\mathrm{fs}}}{\epsilon_{\mathrm{mp}}}}$, and $E_{D A}$ is the energy for data aggregation.

According to the work in [13], heterogeneity in WSN can be classified into three types of heterogeneity: computational heterogeneity, link heterogeneity, and energy heterogeneity. Among the three types of heterogeneity and the most important heterogeneity is the energy heterogeneity because both computational heterogeneity and link heterogeneity will consume more energy resource[13].

We consider three-level energy heterogeneity of sensor nodes as used in SEP-E and DEC. This setting of heterogeneity has no effect on the spatial density of the network [9].

In the three-level of energy heterogeneity networks, there are three types of sensor nodes, i.e., the advanced nodes, intermediate nodes and normal nodes. The intermediate nodes take an initial energy level between that of the advanced nodes and the normal nodes.

As in SEP-E and DEC, the initial energy of the normal nodes is $\mathrm{E}_{0}$, for intermediate nodes, $E_{a d v}=(1+\alpha) E_{0}$ and for advanced nodes, $E_{\text {int }}=(1+\mu) E_{0}$. Where $\alpha$ is the advanced node's energy weight factor and $\mu$ is the energy weight factor for intermediate nodes. The total initial energy of the threelevel energy heterogeneous networks is given by:

$$
E_{\text {total }}=n E_{0}(1+m \alpha+b \mu)
$$

Where $n$ is the number of nodes, $m$ is the proportion of advanced nodes to the total number of nodes $n$ and $b$ is the proportion of intermediate nodes.

\section{PROPOSED SCHEME}

In this section, we outline our suggested scheme Randomized Clustering Algorithm (RCA) which comprise the advantage of a random selection of the $\mathrm{CH}$ along with the effect of the node information on cluster formation (distance to BS, residual energy, and neighbors). In the beginning, we propose our assumptions and then we provide a description of CRA algorithm.

\subsection{Assumptions}

Our approach relies on the following key assumptions regarding the sensor field and sensor nodes:

- $\quad$ Each node has a unique ID to help identify one node from other neighboring nodes.

- All nodes are assumed to be static and randomly distributed.

- The BS has the identification of all nodes in the network $[5,8]$.

\subsection{Scheme descriptions}

The goal of the proposed algorithm is to design a clusteredbased routing protocol that minimizes energy dissipation and maximizes network lifetime of WSN. The proposed algorithm includes two phases, cluster formation phase, and data transmission phase.

\subsubsection{Cluster formation phase}

When the new round is started, the cluster formation phase is initiated. Distance plays an important role while selecting the $\mathrm{CH}$ and since the selection of the $\mathrm{CH}$ is one of the crucial operations in the clustering algorithm. Therefore, selecting the $\mathrm{CH}$ for each cluster should be performed by using minimum communication operation between the sensor nodes and BS.

The calculation of the distance is performed only once in the whole lifetime of the sensor node. This process is initiated by BS by broadcasting the short message which contains the value of its transmitted power and the header to identify that it is from the BS. For generalization, the calculated distance is converted into percentage based distance $d_{\text {percentage }}$ which 
means that the distance of the furthermost node in the deployed area from the BS is represented by 1 and the closest node (i.e. $0 \mathrm{~m}$ from BS itself) is represented as 0 . We set parameter $d_{\text {percentage }}$ to represent the relative distance of nodes as follows

$$
d_{\text {percentage }}=\frac{d_{t o B S}}{\max \left(d_{t o B S}\right)}
$$

where, $d_{t o B S}$ is the distance between sensor node and the BS.

The density of a node $\left(N_{\text {neihbors }}\right)$, is set to represent the number of neighbor nodes located within its transmission range. We set parameter $N_{\text {percentage }}$ to represent the relative density of nodes as follows

$$
N_{\text {percentage }}=\frac{N_{\text {neihbors }}}{N}
$$

Where $N$ is the total number of nodes. The larger $N_{\text {percentage }}$ is, the more neighbors the node has, i.e., it can cover more nodes as a cluster head.

Cluster heads consume more power than other nodes because they have special roles [14]. As a result, it is useful to consider the energy consumption metric in the process of cluster head election and maintain it through the network lifetime.

And since the sensor nodes have energy heterogeneity, RCA also select $\mathrm{CHs}$ according to their energy. Not only is the initial energy capacity $E_{\text {initial }}$ valued, but also its residual energy $E_{\text {residual }}$ after previous rounds of operation. The parameter $E_{\text {percentage }}$ represent the relative residual energy of nodes

$$
E_{\text {percentage }}=\frac{E_{\text {residual }}}{E_{\text {initial }}}
$$

$E_{\text {percentage }}$ indicates that nodes with different initial energy cab be evaluated respectively, which is more suitable for energy heterogeneity.

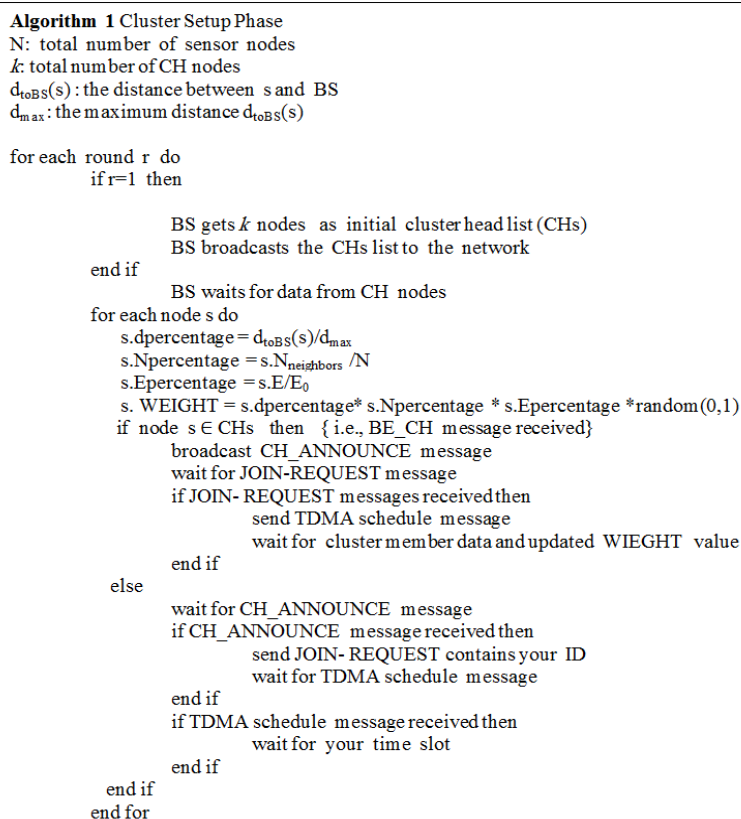

The key idea is that the sensor nodes should perform election of $\mathrm{CHs}$ with respect to the above parameters autonomously. Overall, the goal is to reduce the communication cost and to maximize the network lifetime.

At the beginning of each round $r$ each node $s$ calculates the WEIGHT value as follow:

$$
s . \text { WIEGHT }=E_{\text {percentage }} * d_{\text {percentage }} * N_{\text {percentage }} * Z
$$

Where, $Z$ is a random variable between 0 and 1 .

The WEIGHT value will be used later to determine the nodes that will work as $\mathrm{CHs}$ in round $r+1$. At the end of each round $r$, the $\mathrm{CH}$ node will select the node with the maximum WEIGHT in the current clusters as next $\mathrm{CH}$.

In RCA, at the first $\operatorname{round}(r=1)$, the BS selects the optimal number of $\mathrm{CHs}(k)$ for the network. The BS can take part in the election of the $\mathrm{CHs}$ if and only if the round is the first round. The elected $\mathrm{CHs}$ advertise their role using CSMA just as in $[5,8,9]$. The advertise message (CH_ANNOUNCE ) is a short message containing the node ID of the $\mathrm{CH}$ node.

To ensure concise information is sent to the BS, each node joins and transmits data to the nearest cluster-head. i.e., the non-cluster head nodes choose the $\mathrm{CH}$ that requires minimum communication cost, based on the signal strength of advertisement received and then transmit JOIN-REQUEST message to the chosen $\mathrm{CH}$.

The CHs establish a transmission plan or TDMA schedule, in which, they determine the time for each cluster node where it can transmit its collected data, then transmit a TDMA schedule to the nodes in their cluster. This ensures that there are no collisions among data message and also allows the radio components of non-cluster head nodes to be turned on only during their transmit time $[5,9]$. Once all nodes have known their TDMA schedule, the set-up phase is complete and is followed by the data transmission phase.

The pseudocode of this phase is given in Algorithm 1.

\subsubsection{Data transmission phase}

In this phase, each node sends its data to the respective cluster-head in its allocated time schedule. Just as in DEC, SEP-E, it is assumed that the nodes are synchronized, making all nodes to start the set-up phase at the same time and every node can adjust its transmission power depending on the communication distance or perhaps the received signal strength of the cluster-head advertisement message.

However, in RCA, the data message will contain clustermember WEIGHT's value, by this way, the WEIGHT information of the cluster members (CMs) are known by their respective $\mathrm{CHs}$, thus localized and can be utilized for $\mathrm{CH}$ rotation in the subsequent rounds.

Once the cluster head receives all the data, it performs some signal processing function such as data aggregation. The aggregated data is then forwarded from $\mathrm{CH}$ to the BS.

Before the end of this phase, the current $\mathrm{CHs}$ checks the piggybacked WEIGHT's information received to decide whether they will remain as $\mathrm{CHs}$ or abandon their roles by choosing any cluster member node with the highest WEIGHT as the new $\mathrm{CHs}$.

The pseudocode of this phase is given in Algorithm 2. 


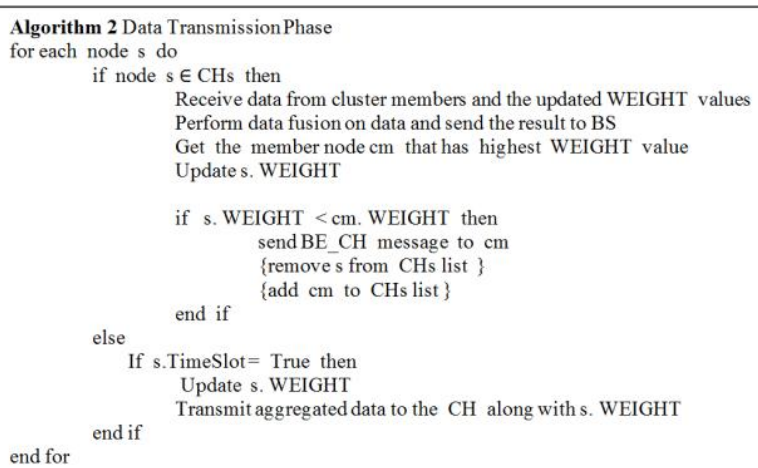

\subsection{Scheme Analysis}

The CHs consume more energy and die too early since they do their role by aggregating and fusing the data traffic generated at the CMs in its cluster and forward it to BS. As a result, in CRA, the nodes with higher weighted value broadcast the CH_ANNOUNCE message. I.e., a node with higher residual energy, with the higher number of neighbors and closer to the base station is more likely to be selected $\mathrm{CH}$ in each round. Moreover, the non-member nodes receiving some $\mathrm{CH}$ _ANNOUNCE message choose the $\mathrm{CH}$ candidate that requires minimum communication cost as its $\mathrm{CH}$ based on the distance between the non-member nodes and the $\mathrm{CH}$ candidates. Therefore, $\mathrm{CH}$ rotation in the subsequent rounds is utilized and the network load is balanced.

If we assumed that $N$ is the total number nodes and $k$ is the number of CHs $(k<<N)$. In RCA, at the beginning of each round, $\mathrm{CH}$ node broadcasts its $\mathrm{CH}$ _ANNOUNCE message, and the total overhead is $\mathrm{k}$. Where after the $\mathrm{CH}$ declaring itself, each non-member node transmits the JOIN-REQUEST message with total overhead equals $(N-k)$. Then, $k \mathrm{CH}$ nodes broadcast TDMA message.

In data transmission phase, cluster member nodes send their data to the corresponding $\mathrm{CH}$ and the total overhead is $\mathrm{N}-k$. Then, the CHs send their data to the BS with total overhead equals $\mathrm{k}$. At the end of this phase $\mathrm{CHs}$ decide to remain $\mathrm{CHs}$ or in the worst case, each $\mathrm{k}$ number of $\mathrm{CHs}$ select any member node to be the subsequent $\mathrm{CH}$. Therefore, the total overhead of this phase is $n-k+k+k$. Consequently, the total message overhead is $(k+(N-k)+k)+(n-k+k+$ $k)=2(k+N)$, whose asymptotic order is $O(N)$.

\section{SIMULATION RESULTS}

In order to evaluate the performance of our proposed algorithm, a simulator has been implemented using MATLAB. In our simulation, 100 sensor nodes are uniformly distributed in a region of size $100 \mathrm{~m} \times 100 \mathrm{~m}, 150 \mathrm{~m} \times$ $150 \mathrm{~m}, 200 \mathrm{~m} \times 200 \mathrm{~m}$, and $250 \mathrm{~m} \times 250 \mathrm{~m}$ on a twodimensional plane.

We use the same energy parameters and radio model that previously described in Section 3 and as discussed in [5,8,12]. The simulated parameters are given in Table 1 .

We define similar performance metrics as used in previous studies [9] [12] [8]. They have defined as:

1) Stability period: the period from the start of the network operation to the first node death.

2) Number of live nodes per round.

3) Half-life: the period from the start of the network to a time when any node uses up half of its energy.
Table1. Setting used in the simulation

\begin{tabular}{|c|c|}
\hline Parameter & Value \\
\hline Network area size & $100 \mathrm{~m} \times 100 \mathrm{~m}$, \\
& $150 \mathrm{~m} \times 150 \mathrm{~m}$, \\
& $200 \mathrm{~m} \times 200 \mathrm{~m}$, and \\
& $250 \mathrm{~m} \times 250 \mathrm{~m}$ \\
\hline Nodes & 100 \\
\hline Initial energy & $0.5 \mathrm{~J}$ to $2.25 \mathrm{~J}$ \\
\hline $\mathrm{E}_{\mathrm{elec}}$ & $50 \mathrm{~nJ} / \mathrm{bit}$ \\
\hline$\epsilon_{\mathrm{fs}}$ & $10 \mathrm{pJ} / \mathrm{bit} / \mathrm{m}^{2}$ \\
\hline$\epsilon_{\mathrm{mp}}$ & $0.00013 \mathrm{pJ} / \mathrm{bit}_{\mathrm{m}} \mathrm{m}^{4}$ \\
\hline $\mathrm{d}_{0}$ & $87 \mathrm{~m}$ \\
\hline $\mathrm{E}_{\mathrm{DA}}$ & $5 \mathrm{~nJ} / \mathrm{bit} / \mathrm{signal}$ \\
\hline Packet size & $4000 \mathrm{bits}$ \\
\hline
\end{tabular}

The algorithms tested using different random topologies. We compared RCA with DEC and SEP-E. As in DEC all algorithms have the same the total energy $(102.5 \mathrm{~J})$ of the network. Table 2 shows the percentage of nodes and their respective energy. Moreover, the optimal parameters of RCA with DEC and SEP-E are utilized to achieve the best performance.

Table 2. Energy setting of sensor nodes.

\begin{tabular}{|c|c|}
\hline$\%$ of nodes & Energy \\
\hline $20 \%$ of the nodes & $2 \mathrm{~J}$ \\
\hline $30 \%$ of the nodes & $1.25 \mathrm{~J}$ \\
\hline $50 \%$ of the nodes & $0.5 \mathrm{~J}$ \\
\hline
\end{tabular}

First, to determine the optimal number of $\mathrm{CH}\left(N_{\text {optimal }}\right)$ for our RCA algorithm, the number of $\mathrm{CHs}$ is varied between 1 and 25 for network size $100 \mathrm{~m} \times 100 \mathrm{~m}$. From figure 1 , note that as the number of $\mathrm{CHs}$ increases from 1 to 10 , the stability period and half-life improve significantly. Both curves are at between 10 and $20 \mathrm{CHs}$ with slight spikes in-between and after $20 \mathrm{CHs}$, the curves start sloping. The optimality of $N_{\text {optimal }}$ lies around $17 \mathrm{CHs}$. This due to when there are few $\mathrm{CHs}$, CMs will often transmit data large distances to their $\mathrm{CHs}$, as a result, this exhaust their energy faster.

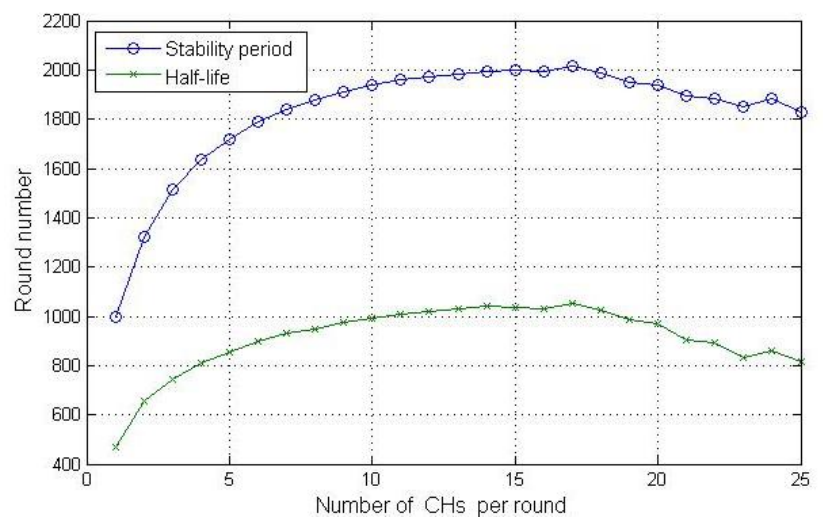

Figure 1: Stability period and Half-life in our algorithm as the number of $\mathrm{CH}$ varies from 1 to 25 . 
If there are additional $\mathrm{CHs}$ (from 10 to 20), $\mathrm{CM}$ nodes can easily locate a nearby $\mathrm{CH}$. But after $20 \mathrm{CHs}$, there is not much local data aggregation being performed. Extra energy will be expended due to the smaller sizes of the clusters formed. It is concluded that our algorithm is stable when the number of $\mathrm{CH}$ varies between 10 to 20 .

For further analysis, we investigate other performance metrics such as stability period, Half-life and the number of live nodes per round.

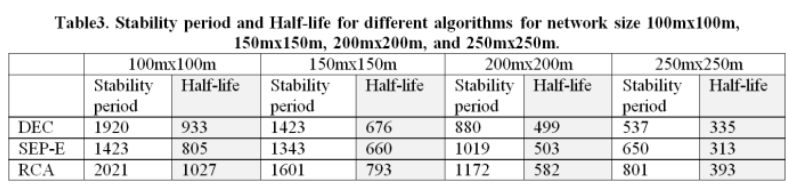

Table 3 shows some significant results of our algorithm for different network size. Overall, our algorithm improves the WSN lifetime compared with DEC and SEP-E up to a magnitude of $6 \%$ and $42 \%$ respectively for network size $100 \mathrm{~m} \times 100 \mathrm{~m}$. As the network size increased to $250 \mathrm{~m} \times 250 \mathrm{~m}$ our algorithm improves the WSN stability period compared with DEC and SEP-E up to a magnitude of $49 \%$ and $23 \%$ respectively.

From Figure 2, we notice that the stability period for all algorithm decreased as network size increased this is expected because the distance between nodes increased and more energy is required to aggregate and send data to the BS. Moreover, DEC improves the WSN stability period compared with SEP-E up to a magnitude of $35 \%$ for network size $100 \mathrm{~m} \times 100 \mathrm{~m}$, while as the network size increased the magnitude decreased for DEC and increased for SEP-E up to a magnitude of $21 \%$ compared with DEC for network size $250 m \times 250 m$. Furthermore, our RCA algorithm improves the WSN Half-life compared with DEC and SEP-E up to a magnitude of $10 \%$ and $28 \%$ respectively as shown in figure 2 .
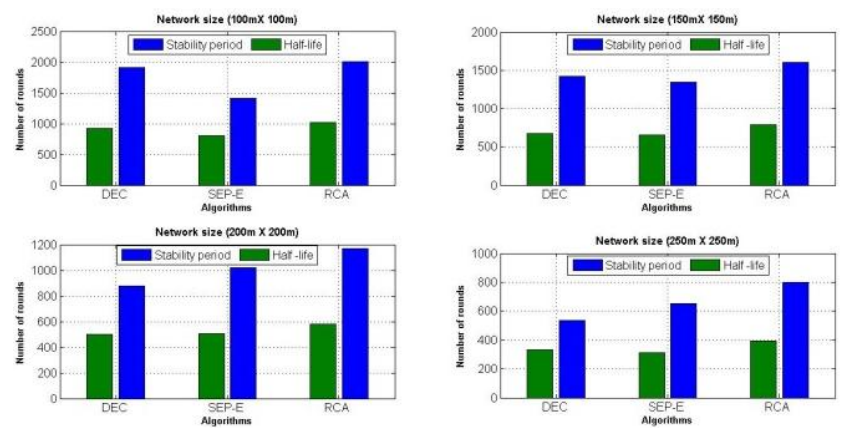

Figure 2: Stability period and Half-life for different network size.

Figure 3, show the number of live nodes per round for different network size. It is noted that for network size $100 m \times 100 m$ the curve of SEP-E drops slowly until the end of the network because SEP-E protocol adapts slowly with heterogeneity. While RCA and DEC curves gradual descent at the beginning is due to the different energy levels of the nodes in the network at the beginning. Moreover, the stability of our algorithm is better than DEC and SEP-E for different network size. However, as the network size increased the stability of SEP-E improved, while the stability of DEC decreased as shown in figure 3 .
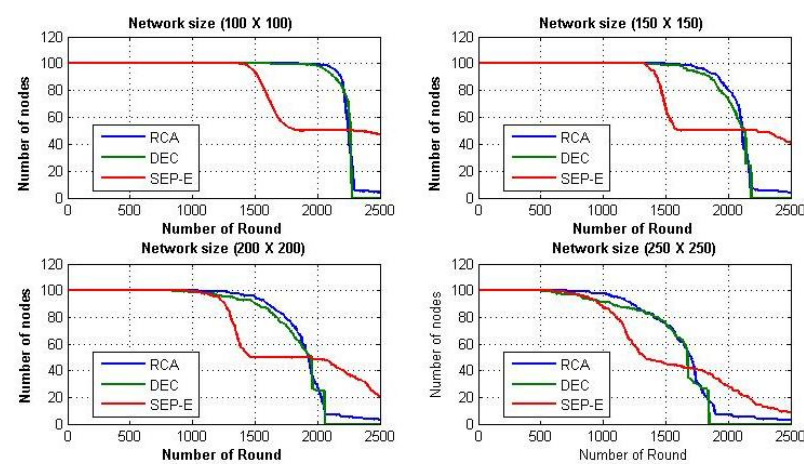

Figure 3: Number of alive nodes per round for different network size.

Further, the average energy consumption per round of the network was calculated by changing the size of the network area. i.e. $(100 \times 100),(150 \times 150),(200 \times 200)$ and $(250 \times 250)$.

Average energy consumption per round until the death of the first sensor node is equal to the total energy consumed until the death of the first node divided by the total number of rounds until the death of the first node in the sensor network. In this comparative parameter, the most efficient algorithm is that with the lowest average energy consumption per round, which determines a longer life of the sensor network.

In Figure 4 the average energy consumption per round is shown for RCA, DEC, and SEP-E algorithms. The average energy consumption for RCA is $2 \%, 5 \%, 9 \%, 21 \%$ times less compared to DEC and $21.5 \%, 12 \%, 8.5 \%, 8 \%$ times less compared to SEP-E for network size $100 \mathrm{~m} \times 100 \mathrm{~m}$, $150 m \times 150 m, 200 m \times 200 m$, and $250 m \times 250 m$, respectively. Note that as the network size increased the average energy consumption per round for SEP-E and RCA increased slightly, while there is a remarkable increase for DEC.

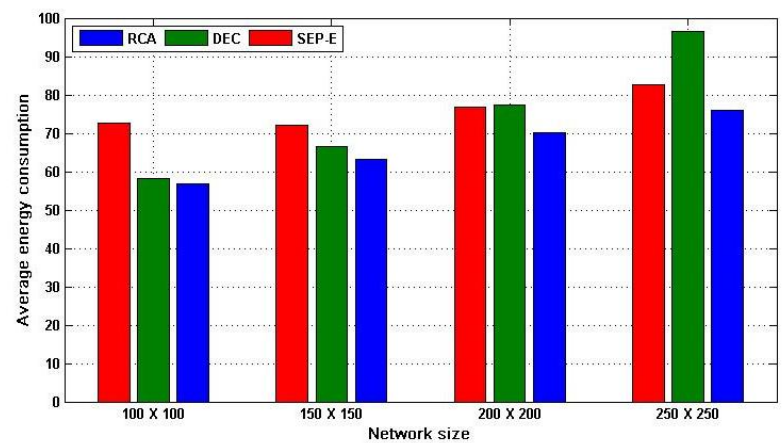

Figure 4: Average energy consumption per round versus network size for RCA, DEC and SEP-E algorithms

The proposed scheme clearly outperforms the others with respect to the network lifetime. Observe that our scheme selects the $\mathrm{CHs}$ based on a random approach as well as based on deterministic parameters, which gives desirable properties for our scheme. In proposed scheme the election process of $\mathrm{CH}$ is locally decided based on each node's information, moreover the scheme guarantees that every node has a chance of election as $\mathrm{CH}$ and ensures a fixed number of $\mathrm{CHs}$ is chosen as in deterministic approach (e.g. DEC) and unlike in probabilistic approaches ( e.g. SEP-E) each round is independent of the next round. 


\section{CONCLUSIONS}

In this paper, we have proposed an algorithm called RCA. Like SEP-E, RCA also implements randomization but with the addition of local information parameters as in DEC. CRA divide the three-level energy heterogeneity of sensor network into a two-level hierarchical network. The CRA algorithm uses distributed scheme for $\mathrm{CH}$ selection and cluster formation. Based on proposed study RCA improves the network lifetime of WSNs when compared with the threelevel energy heterogeneity based algorithms i.e., DEC and SEP-E. Overall, RCA algorithm improves the WSN lifetime compared with DEC and SEP-E up to a magnitude of $6 \%$ and $42 \%$ respectively for network size $100 \mathrm{~m} \times 100 \mathrm{~m}$. Furthermore, RCA algorithm improves the WSN Half-life compared with DEC and SEP-E up to a magnitude of $10 \%$ and $28 \%$ respectively. Besides, it is notice that as the network size increased the average energy consumption per round for SEP-E and RCA increased slightly, while there is a remarkable increase for DEC.

\section{REFERENCES}

[1] Jennifer Yick, Biswanath Mukherjee, Dipak Ghosal, Wireless sensor network survey, The International Journal of Computer and Telecommunications Networking, Vol.52, no.12, pp. 2292-2330, 2008.

[2] Yueh-Min Huang, Meng-Yen Hsieh, Frode Eika Sandnes, Wireless sensor networks: A survey, in: Advanced Information Networking and Applications Workshops, WAINA '09. International Conference on, pp. 636-641, 2009.

[3] Karl,H.,Willig,A.: Protocols and Architectures for Wireless Sensor Networks. Wiley, England (2007).

[4] Khediri, S.E., Nasri, N., Wei, A., Kachouri, A., A new approach for clustering in wireless sensors networks based on LEACH. Procedia Comput. Sci. 32, 1180-1185 (2014).

[5] Wendi Rabiner Heinzelman, Anantha Chandrakasan, and Hari Balakrishnan, Energy-efficient communication protocol for wireless microsensor networks, Proceedings of the 33rd Hawaii International Conference on System Sciences, 2000, pp. 1-10.

[6] Wendi Rabiner Heinzelman, Anantha Chandrakasan, and Hari Balakrishnan, An application-specific protocol architecture for wireless microsensor networks. IEEE
Transactions on Wireless Communications, 1, pp.660 670, 2002.

[7] M. J. Handy, M. Haase and D. Timmermann, Low energy adaptive clustering hierarchy with deterministic cluster-head selection, 4th International Workshop on Mobile and Wireless Communications Network, 2002, pp. 368-372.

[8] Aderohunmu, F.A., Deng, J.D. and Purvis, M.K. , A Deterministic Energy efficient Clustering protocol for wireless sensor networks. In Proceedings of the Seventh IEEE International Conference on Intelligent Sensors, Sensor Networks and Information Processing (IEEEISSNIP), pp. 341-346, Dec. 2011. Adelaide, Australia.

[9] Aderohunmu, F.A., Deng, J.D. and Purvis, M.K., Enhancing clustering in wireless sensor networks with energy heterogeneity. Inter. Journal of Business Data Communications and Networking, 7(4), pp. 18-32, 2011.

[10] Li Qing, Qingxin Zhu, Mingwen Wang, Design of a distributed energy-efficient clustering algorithm for heterogeneous wireless sensor networks, Computer Communications, Volume 29, Issue 12, 4 August 2006, pp. 2230-2237.

[11] Mainak Chatterjee, Sajal K. Das, and Damla Turgut. 2002. WCA: A Weighted Clustering Algorithm for Mobile Ad Hoc Networks. Cluster Computing 5, 2, pp. 193-204, 2002.

[12] G. Smaragdakis, I. Matta, and A. Bestavros. SEP: A Stable Election Protocol for clustered heterogeneous wireless sensor networks. In Proceeding of the International Workshop on SANPA, 2004.

[13] Dilip Kumar, Trilok C. Aseri, R.B. Patel, EEHC: Energy efficient heterogeneous clustered scheme for wireless sensor networks, Computer Communications, Volume 32, Issue 4, 4 March 2009, pp. 662-667, ISSN 01403664.

[14] Manar A. Mizher, Saleh H. Al-Sharaeh, Mel Choo Ang, Ayman M. Abdalla, Manal A. Mizher, Centroid dynamic sink location for clustered wireless mobile sensor networks, Journal of Theoretical and Applied Information Technology 73 (3), pp. 481-491, 2015.

[15] B. Mamalis, D. Gavalas, C. Konstantopoulos, and G. Pantziou, Clustering in wireless sensor networks, RFID and Sensor Networks: Architectures, Protocols, Security and Integrations, pp. 324-353, 2009. 